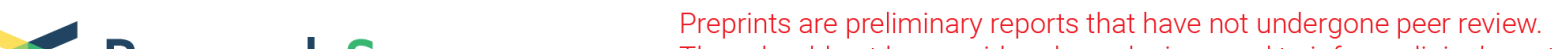 Research Square
or referenced by the media as validated information.
}

\section{Clinical value of peripheral blood monocytes related indicators alone or in combination with T-SPOT.TB in the diagnosis of smear negative pulmonary tuberculosis}

\section{Xiali Lyu}

Beijing Tuberculosis and Thoracic Tumor Research Institute

\section{Saisai Wang}

Beijing Tuberculosis and Thoracic Tumor Research Institute

\section{Hongyan Jia}

Beijing Tuberculosis and Thoracic Tumor Research Institute

\section{Zihui Li}

Beijing Tuberculosis and Thoracic Tumor Research Institute

\section{Wei Shu}

Beijing Tuberculosis and Thoracic Tumor Research Institute

\section{Wanli Kang}

Beijing Tuberculosis and Thoracic Tumor Research Institute

\section{Liping Pan}

Beijing Tuberculosis and Thoracic Tumor Research Institute

\section{Zongde Zhang}

Beijing Tuberculosis and Thoracic Tumor Research Institute

\section{Qi Li ( $D$ lq0703@hotmail.com )}

Beijing Tuberculosis and Thoracic Tumor Research Institute

\section{Research article}

Keywords: Smear negative pulmonary tuberculosis, SNPT, ML ratio, T-SPOT.TB, Diagnosis accuracy, Misdiagnosis rate

Posted Date: December 19th, 2019

DOI: https://doi.org/10.21203/rs.2.19299/v1

License: (c) (i) This work is licensed under a Creative Commons Attribution 4.0 International License. Read Full License 


\section{Abstract}

Background To improve the early diagnosis of SNPT, is one of the most important measures to reduce the incidence of pulmonary tuberculosis in China. This paper studied the expression characteristics of monocytes and other indicators in peripheral blood of SNPT, and discussed the clinical value of those indicators alone or in combination with T-SPOT.TB in the diagnosis and differential diagnosis of SNPT.

Methods : We collected 134 SNPT patients, 56 lung cancer patients, 95 pneumonia patients (including 51 CAP patients and $44 \mathrm{HAP}$ patients) and $117 \mathrm{HC}$ as research subjects from Beijing Chest Hospital, Capital Medical University from January, 2018 to December, 2018. The relevant indicators of peripheral blood monocytes alone or in combination with T-SPOT.TB of the research subjects were analyzed. Data were analyzed by SPSS 25.0 software. Measurement data were analyzed by $t$ test or $z$ test. Enumeration data were analyzed by Chi-square test. ROC curve and diagnostic accuracy were used to evaluate the clinical value.

Results : The percentage of monocytes, ML ratio and the positive rate of T-SPOT.TB in SNPT group were higher than those in heathy control group, lung cancer group and pneumonia group, with statistically significance $(P<0.05)$. In comparison with healthy control group, the sensitivity $(98.51 \%)$ and accuracy (80.88\%) of ML ratio combined with T-SPOT.TB (parallel test) for the diagnosis of SNPT group were increased and the rate of missed diagnosis (1.49\%) was decreased. In the differential diagnosis of SNPT and lung cancer, the percentage of monocytes was better than the other three indicators, and the combined detection with T-SPOT.TB ( series test) can reduce the misdiagnosis rate and improve the diagnosis accuracy. In the differential diagnosis of SNPT and pneumonia, percentage of monocytes and ML ratio combined with T-SPOT.TB (series test) can reduce the misdiagnosis rate and improve the diagnosis accuracy.

Conclusions: The percentage of monocytes and ML ratio have good value of early auxiliary diagnosis, and the combined diagnosis indicators can make up for the deficiency of T-SPOT.TB in the diagnosis of active tuberculosis, and improve the early diagnosis rate of SNPT and the clinical value of differential diagnosis.

\section{Background}

Smear negative pulmonary tuberculosis (SNPT) refers to pulmonary tuberculosis with sputum acidfasting staining negative[1]. In 2010, the fifth national tuberculosis epidemiological survey showed that the prevalence of SNPT among people aged 15 and above was $393 / 100,000$, accounting for $85.65 \%$ of active tuberculosis patients[2]. In comparison with 2005, the incidence of SNPT in China in 2014 was increased by $24.2 \%$, and the incidence of SNPT was about 5.9 times higher than that of smear positive pulmonary tuberculosis[3]. All these suggest that SNPT patients are the main target of active tuberculosis. 
The existence of SNPT involves a variety of reasons, including the sensitivity of existing detection methods for Mycobacterium tuberculosis, the characteristics of the lesion of the patients, the quality of sputum retention of the patients, the quality of sputum test in the laboratory, etc. Even when sputum Mycobacterium tuberculosis nucleic acid test was used, the detection rate was lower. The sensitivity of Xpert MTB/RIF test in the diagnosis of SNPT was 67\% (62\% 72\%)[4]. Therefore, it is particularly important to find a simple and easy diagnostic indicator with high sensitivity and specificity for the early diagnosis of SNPT.

Monocytes are the precursor of macrophages, which are the target cells of Mycobacterium tuberculosis and play an important role in the occurrence and development of tuberculosis. The percentage of monocytes is one of the indicators of blood routine test. The ratio of monocytes to lymphocytes (ML ratio) in active tuberculosis patients was significantly higher than that in healthy control, and was closely related to the severity of the lesion[5]. This study retrospectively analyzed the expression characteristics of peripheral blood monocytes, ML ratio and other indicators in SNPT patients, and evaluated the clinical value of those indicators alone and in combination with T-SPOT.TB in the diagnosis and differential diagnosis of SNPT, so as to provide a simple, feasible and new indicator for the early diagnosis of SNPT.

\section{Methods}

\section{Study participants}

This retrospective study analyzed 402 participants including healthy control and patients who were hospitalized in Beijing Chest Hospital, Capital Medical University, Beijing, China, and the data were accessed from January 1, 2018 to December 31, 2018.

(a) Smear negative pulmonary tuberculosis (SNPT) group: 134 patients (87 men, accounting for $64.90 \%$; 47 women, accounting for $35.10 \%$; mean age $37.05 \pm 13.61$ years).

(b) Lung cancer group: 56 patients (23 men, accounting for $41.10 \%$; 33 women, accounting for $58.90 \%$; mean age $54.27 \pm 6.30$ years).

(c) Pneumonia group: 95 patients (including 51 patients diagnosed with community-acquired pneumonia, CAP and 44 patients diagnosed with hospital-acquired pneumonia, HAP) (62 men, accounting for 65.30\%; 33 women, accounting for $34.70 \%$; mean age $45.76 \pm 11.70$ years).

(d) Healthy control (HC) group: 117 participants (27 men, accounting for $23.10 \%$; 90 women, accounting for $76.90 \%$; mean age $33.84 \pm 9.96$ years).

The gender composition and age of HC group were statistically different from that of SNPT group, lung cancer group and pneumonia group $(P<0.05)$. In addition, the age of SNPT group was significantly lower than that of lung cancer group and pneumonia group $(P<0.05)$.

\section{Inclusion and exclusion criteria}




\section{The inclusion criteria were as follows:}

(1) SNPT group: a. The diagnosis of the patients conforms to the diagnostic criteria for SNPT in the Health Industry Standards of the People's Republic of China - "Diagnosis of pulmonary tuberculosis (WS 288-2017)" and "Classification of tuberculosis (WS 196-2017)". b. Age 18 60 years, men and women. c. The initial and recurrent patients were included. $d$. Clinical data were complete.

(2) Lung cancer group: a. The diagnosis of the patients conforms to the diagnostic criteria in the Health Industry Standards of the People's Republic of China - "Specification for diagnosis and treatment of primary lung cancer (2018)". b. Age 18 60 years, men and women. c. All patients were initial diagnosis and before chemoradiotherapy. $d$. Clinical data were complete.

(3) CAP and HAP group: a. The diagnosis of the patients respectively conforms to the diagnostic criteria by the Chinese society of respiratory medicine - " Guidelines for diagnosis and treatment of communityacquired pneumonia in adults in China (2016)" and by the infectious diseases group, the Chinese society of respiratory medicine - " Guidelines for diagnosis and treatment of hospital-acquired pneumonia and ventilator-associated pneumonia in adults in China (2018)". b. Age 18 60 years, men and women. c. All patients were pre-treatment. d. Clinical data were complete.

(4) HC group: a. All participants were with negative T-SPOT.TB, normal chest imaging, no clinical symptoms of active tuberculosis or other chronic diseases. b. Age 18 60 years, men and women. c. The relevant data were complete.

The exclusion criteria were as follows: (1) Patients identified as non-tuberculous mycobacterium (NTM) disease. (2) Pulmonary tuberculosis with lung cancer. (3) Patients with abnormal immune function, HIV infection, hematologic disease, serious liver and kidney function impairment, pregnancy or lactation. (4) Recent application of immunosuppressive. (5) Incomplete clinical data.

\section{Detection methods}

Blood routine test: The inpatients in the morning of the next day and healthy control on the day of the physical examination were respectively taken $6 \sim 8 \mathrm{ml}$ heparin anticoagulant whole blood, and the whole blood cell count was performed by the blood cell analyzer.

T-SPOT.TB examination: The inpatients in the morning of the next day and healthy control on the day of the physical examination were respectively taken $6 \mathrm{ml}$ peripheral blood, and T-SPOT.TB test kit (Oxford Immunotech, UK) was applied for detection. The peripheral blood mononuclear cell (PBMC) was prepared to $2.5 \times 10^{6} / \mathrm{ml}$ cell suspensions by resuspending and mixing with $5 \mathrm{ml}$ RPM1640 medium. $100 \mu \mathrm{l}$ cell suspensions were added to each well in the pre-coated plate with anti- IFN-y antibody, and 4 wells were set for each case: negative control, positive control, antigen A (early secretory antigen target-6, ESAT-6) and antigen $B$ (culture filtrate protein-10, CFP-10) wells. Then, it was incubated in an incubator containing $5 \% \mathrm{CO}_{2}$ at $37^{\circ} \mathrm{C}$ for $16-24 \mathrm{~h}$, washed with PBS, added with biotin-labeled secondary antibodies, stained by 
enzyme, and counted through the enzyme-linked immune spot system (CTL-Immu-noSpot@S5 Versa Analyzer).

\section{Observe and analysis the indicators and its limit value}

Patients information: general demographics (age, sex).

Blood routine test: Percentage of monocytes (Percentage of M), percentage of lymphocytes (Percentage of $L), M L$ ratio ( $M L$ ratio= percentage of $M /$ percentage of $L)$.

T-SPOT.TB examination: The test result is "reactive", with reference to the following criteria: when the number of negative control hole spots is $0 \sim 5$, and (the number of antigen $A$ or $B$ hole spots) minus (the number of negative control hole spots) $\geq 6$; When the number of negative control hole spots is $6-10$, and (the number of antigen A or B hole spots) $\geq 2$ * (the number of negative control hole spots). If the above criteria are not met and the positive control hole is normal, the test result is "non-reactive".

\section{Statistical analysis}

The data were analyzed by SPSS 25.0 software. The measurement data in normal distribution were expressed as mean \pm standard deviation ( $x \pm s$ ) and analyzed by $t$ test, while the measurement data in non-normal distribution were expressed as median (interquartile range) $[M(P 25 \sim P 75)]$ and analyzed by $Z$ test. The enumeration data were expressed as percentage and analyzed by Chi-square. When the theoretical frequency $<1$, the Fisher exact probability test was used. The receiver operating characteristic (ROC) curve and diagnostic accuracy were used to evaluate the clinical value. $P<0.05$ was considered statistically significant. Clinical diagnostic efficacy of parallel test and series test: Sensitivity = true positive cases/(true positive cases + false negative cases); Specificity = true negative cases/(true negative cases + false positive cases); Missed diagnosis rate $=1$ - sensitivity; Misdiagnosis rate $=1$ specificity; Diagnostic accuracy $=$ (true positive cases + true negative cases $) /($ true positive cases + false positive cases + true negative cases + false negative cases).

\section{Results}

\section{Expression of monocytes related indicators and T-SPOT.TB in SNPT patients}

Table 1 showed the results of peripheral blood monocytes, lymphocytes, ML ratio, T-SPOT.TB and other indicators in SNPT patients. As shown in table 1, the percentage of $M, M L$ ratio and the positive rate of TSPOT.TB in SNPT group were significantly higher than those in HC group, lung cancer group and pneumonia group $(\mathrm{P}<0.05)$. In addition, in comparison with $\mathrm{HC}$ group, the percentage of $\mathrm{L}$ in lung cancer group and pneumonia group were significantly decreased, $\mathrm{ML}$ ratio and the positive rate of T-SPOT.TB were significantly increased $(P<0.05)$. 
The ROC curve of four indicators of 134 SNPT patients was shown in figure 1, and the prediction probability was shown in table 2. As shown in figure 1 and table 2, the AUC curve of single indicator were 0.688 0.952, and the order from high to low was T-SPOT.TB, ML ratio, percentage of $L$ and percentage of M. Parallel test was used for combined detection, and the AUC curve was 0.963 0.975, and the order from high to low was ML ratio combined with T-SPOT.TB, percentage of M combined with T-SPOT.TB, percentage of $L$ combined with T-SPOT.TB.

The mean value of three indicators in $\mathrm{HC}$ group (Namely: percentage of $M>7.62 \%$, percentage of $\mathrm{L}<34.76 \%, \mathrm{ML}$ ratio $>0.23 \%$ ) and T-SPOT.TB $(\mathrm{A}$ or $\mathrm{B}) \geq 6$ were taken as the evaluation limits. The evaluation results of its clinical diagnostic efficacy in the diagnosis of SNPT alone or combined detection were shown in table 3. As shown in table 3 , the sensitivity (80.6\%), specificity $(60.68 \%)$ and diagnostic accuracy $(71.31 \%)$ of $M L$ ratio were higher than percentage of $M$ and percentage of $L$, but lower than $T-$ SPOT.TB. In comparison with single indicator, the sensitivity (98.51\%) and diagnostic accuracy (80.88\%) of $\mathrm{ML}$ ratio combined with T-SPOT.TB (parallel test) were increased, while the rate of missed diagnosis was decreased (1.49\%).

\section{Analysis of the differential diagnostic value of monocytes related indicators and T-SPOT.TB in SNPT, lung cancer and pneumonia patients}

The differential diagnostic value of monocytes related indicators in SNPT and lung cancer patients: The mean value of three indicators in HC group (Namely: percentage of $M>7.62 \%$, percentage of $L<34.76 \%$, $\mathrm{ML}$ ratio $>0.23 \%$ ) and T-SPOT.TB ( $\mathrm{A}$ or $\mathrm{B}) \geq 6$ were taken as the evaluation limits. The evaluation results of its clinical diagnostic efficacy in the diagnosis of SNPT and lung cancer alone or combined detection were shown in table 4 . As shown in table 4 , the specificity $(78.57 \%)$ and diagnostic accuracy $(72.63 \%)$ of percentage of $M$ were higher than percentage of $L$ and $M L$ ratio. Although its sensitivity and diagnostic accuracy were slightly lower than T-SPOT.TB, its specificity was higher than T-SPOT.TB and its misdiagnosis rate was lower than T-SPOT.TB. In comparison with single diagnosis indicator, the sensitivity $(59.70 \%)$ of percentage of M combined with T-SPOT.TB (series test) was decreased, while the specificity (91.07\%) was significantly increased, and the misdiagnosis rate (8.93\%) was significantly decreased.

The differential diagnostic value of monocytes related indicators in SNPT and pneumonia patients: The mean value of three indicators in $\mathrm{HC}$ group (Namely: percentage of $M>7.62 \%$, percentage of $L<34.76 \%$, $\mathrm{ML}$ ratio $>0.23 \%$ ) and T-SPOT.TB ( $\mathrm{A}$ or $\mathrm{B}) \geq 6$ were taken as the evaluation limits. The evaluation results of its clinical diagnostic efficacy in the diagnosis of SNPT and pneumonia alone or combined detection were shown in table 5 . As shown in table 5 , the sensitivity $(80.6 \%)$ and diagnostic accuracy $(65.07 \%)$ of $M L$ ratio were higher than percentage of $L$ and percentage of $M$, while the missed diagnosis rate $(19.40 \%)$ was lower than percentage of $L$ and percentage of $M$. In comparison with single diagnosis indicator, the sensitivity $(69.40 \%)$ of ML ratio combined with T-SPOT.TB (series test) was slightly decreased, while the specificity $(75.79 \%)$ was significantly increased, the misdiagnosis rate $(24.21 \%)$ was decreased, and the diagnostic accuracy rate $(72.05 \%)$ was increased. 


\section{Discussion}

Diagnostic methods of SNPT include sputum Mycobacterium tuberculosis culture and nucleic acid testing, imaging and immunological test (including tuberculosis skin test (TST), tuberculosis antibody (TB-Ab) and interferon- $\gamma$ release assays (IGRAs)). Among them, sputum Mycobacterium tuberculosis culture, as the gold standard for the diagnosis of tuberculosis, has high specificity but low sensitivity, and the culture time is long, leading to a lag in the diagnosis. Even if sputum Mycobacterium tuberculosis nucleic acid test was used, the detection rate was relatively low. The sensitivity of Xpert MTB/RIF test in the diagnosis of SNPT was $67 \%(62 \% \sim 72 \%)$ [4]. Chest imaging examination is an important basis for the diagnosis of SNPT, but the sensitivity of chest radiographs was $81.6 \%[6]$. Immunological test is one of the important auxiliary diagnostic evidences for SNPT[7]. However, the sensitivity of immunological detection also failed to meet expectations. Table 1 of this paper also showed that the positive rate of TSPOT.TB in SNPT group was $87.3 \%$, which is close to that reported in the literature[8]. The specificity of TSPOT.TB is low as shown in table 1 of this paper. The positive rate of T-SPOT.TB in lung cancer group and pneumonia group respectively reached to $32.10 \%$ and $36.80 \%$, which suggested that it had low specificity in the differential diagnosis of SNPT and non-pulmonary tuberculosis of pulmonary diseases and was prone to misdiagnosis. Therefore, T-SPOT.TB has high diagnostic value for active tuberculosis in countries with low burden of tuberculosis [9]. However, in countries with high burden of tuberculosis, it is controversial whether T-SPOT.TB can be used as the diagnostic basis of active tuberculosis because of its sensitivity and specificity, since it is unable to effectively distinguish between active tuberculosis and Mycobacterium tuberculosis latent infection[10].

Monocytes are the target cells of Mycobacterium tuberculosis, and lymphocytes are the main effector cells of tuberculosis immunity[11]. The level of ML ratio can reflect the immune status of the body to Mycobacterium tuberculosis infection, and clinical detection is simple and easy. Among them, ML ratio is considered to be an important indicator determining the immune efficiency of host in the state of Mycobacterium tuberculosis infection, which is closely related to monocytes and lymphocytes, and is easy to be quantified in peripheral blood test. ML ratio was related to the growth of Mycobacterium tuberculosis, suggesting that ML ratio could reflect the occurrence and development of tuberculosis[11]. $\mathrm{ML}$ ratio of active pulmonary tuberculosis patients was significantly higher than that of healthy control, and was closely related to the severity of lesions[5]. In comparison with healthy control, latent infection individuals and cured tuberculosis patients, active tuberculosis patients had higher ML ratio and lower $M L$ ratio after anti-tuberculosis treatment[12]. These studies suggest that $M L$ ratio has certain clinical value in the diagnosis of active tuberculosis, evaluation of disease severity, and monitoring of antituberculosis efficacy[12-16].

As shown in table 1, percentage of M, ML ratio and positive rate of T-SPOT. TB in SNPT group were higher than those in healthy control group, lung cancer group and pneumonia group. This was consistent with the reports of Rakotosamimanana[17] and Sampath Pavithra[5]. The results showed that Mycobacterium tuberculosis infection could lead to abnormal changes in the "quality" and "quantity" of host immune cells, that is, monocytes, the precursor of macrophages, were increased to engulf the growing and 
reproducing Mycobacterium tuberculosis. The abnormal immune response caused by Mycobacterium tuberculosis and host malnutrition may inhibit or affect the differentiation and aggregation of lymphocytes. It is suggested that percentage of $M$ and $M L$ ratio have potential value in the diagnosis of active pulmonary tuberculosis, especially SNPT. As shown in Fig. 1 and Table 2, in comparison with healthy control, the AUC curve of percentage of M and ML ratio of SNPT patients respectively reached to 0.688 and $0.791(P=0.000)$, suggesting that these indicators have auxiliary diagnostic significance for SNPT. The AUC curve of percentage of M and ML ratio combined with T-SPOT.TB (parallel test) respectively increased to 0.965 and 0.975 , suggesting that the combined detection of indicators is helpful to improve the early detection rate of SNPT. The mean value of healthy control group (table 1) was taken as the evaluation limits. As shown in Table 3, the sensitivity of percentage of $M$ and $M L$ ratio respectively was $70.15 \%$ and $80.60 \%$, which was slightly lower than T-SPOT.TB (87.31\%). However, the combined detection (parallel test) can improve the sensitivity and decrease missed diagnosis rate. It is suggested that percentage of $M$ and $M L$ ratio have good value of early auxiliary diagnosis, and the combined diagnosis of indicators can make up for the deficiency of T-SPOT.TB in the diagnosis of active tuberculosis, and improve the early diagnosis rate of SNPT. In addition, the specificity of percentage of M and ML ratio of SNPT patients in this paper were lower than that of T-SPOT.TB, because T-SPOT.TB in healthy control group were all negative. If the selected control group contains latent infection of Mycobacterium tuberculosis, the specificity of these two indicators may be close to T-SPOT.TB, as reported that the specificity of IGRAs was $60 \sim 90 \%$ in the literature[10].

Lung cancer and pneumonia are relatively high incidence of respiratory diseases, but also are common diseases of differential diagnosis of SNPT. Because part of the imaging characteristics of lung cancer (such as soft tissue density of piece of shadow, nodular shadows, empty shadow, etc), respiratory symptoms (such as cough, hemoptysis, etc) are similar to SNPT patients. Part of the image characteristics of pneumonia (such as patch, consolidation, nodules, sprouts, etc) and infections, respiratory symptoms (such as fever, cough, sputum, etc) are also similar to SNPT patients. Therefore, in the case of negative bacteriology, it is necessary to seek other relevant indicators for differential diagnosis, in order to improve the diagnosis rate and reduce the misdiagnosis rate.

In terms of differential diagnosis between SNPT patients and lung cancer patients, as shown in Table 4, in comparison with ML ratio and T-SPOT.TB, the specificity of percentage of M was higher and up to $78.57 \%$, and the misdiagnosis rate $(21.43 \%)$ was lower. The specificity of percentage of $\mathrm{M}$ combined with T-SPOT.TB (series test) increased to $91.07 \%$ and the misdiagnosis rate reduced to $8.93 \%$. It is suggested that monocytes increasing is an important immunological feature of pulmonary tuberculosis, which is related to the invasion, growth, reproduction and cytokine release of Mycobacterium tuberculosis. While cellular immune response plays an important role in the occurrence and development of tumors, macrophages as tumor lysis cells may not be the main effector cells[18]. As shown in table 1 in this paper, the percentage of $\mathrm{M}$ in lung cancer group is close to that in healthy control group. Therefore, in the differential diagnosis of SNPT and lung cancer, percentage of M has good differential diagnosis value, which provides a basis for improving the diagnosis rate of SNPT. Although the sensitivity of ML ratio was higher than percentage of $\mathrm{M}$, its specificity was lower (41.07\%) and the misdiagnosis rate was higher 
(58.93\%). Even when ML ratio combined with T-SPOT.TB, the specificity and misdiagnosis rate were not better than percentage of $\mathrm{M}$. In addition, although T-SPOT.TB is highly sensitive, the latent infection rate of Mycobacterium tuberculosis in China is high[19], which leads to low specificity (67.86\%) and high misdiagnosis rate $(32.14 \%)$ in the differential diagnosis of SNPT and lung cancer.

In terms of differential diagnosis between SNPT patients and pneumonia patients, as shown in Table 5, although the sensitivity of percentage of $M$ and $M L$ ratio was high $(70.15 \%$ and $80.60 \%)$, the specificity of two indicators was low ( $46.32 \%$ and $43.16 \%)$, leading to high misdiagnosis rate $(53.68 \%$ and $56.86 \%)$ in the differential diagnosis of SNPT and pneumonia. This may be similar to the immune response that induced by partial pneumonia, which can both induce phagocytosis and killing of pathogens by macrophages, leading to the increase of peripheral blood monocytes at different degrees. At the same time, chemotaxis inducing by pulmonary inflammation can cause neutrophils to move to the inflammatory site, resulting in neutrophils increasing in peripheral blood, percentage of $L$ decreasing, and $M L$ ratio increasing, which is not significantly different from ML ratio of SNPT patients. In addition, even if T-SPOT.TB was applied to differentiate SNPT and pneumonia, its misdiagnosis rate was higher (36.84\%). Combined detection of percentage of $\mathrm{M}$ and $\mathrm{ML}$ ratio with T-SPOT.TB (series test) can improve the specificity, respectively reaching to $76.84 \%$ and $75.79 \%$, thus respectively reducing the misdiagnosis rate to $23.16 \%$ and $24.21 \%$ in the differential diagnosis of SNPT and pneumonia.

There were limitations in our study: there were differences in age and gender between groups, for example, the incidence of primary lung cancer was relatively advanced age. Mainly because this was a retrospective study, the age and gender of study participants could not be completely controlled. However, considering that percentage of $\mathrm{M}$ and $\mathrm{ML}$ ratio were not affected by age and gender, this inter-group differences would not have a significant impact on the research results.

\section{Conclusions}

In conclusion, the percentage of $\mathrm{M}$ and $\mathrm{ML}$ ratio in peripheral blood are highly expressed in SNPT patients, which is cheap and easy to measure, and can be used as auxiliary diagnostic indicators for SNPT. In the differential diagnosis of SNPT and lung cancer, the percentage of M is better than the other three indicators, and its combined detection with T-SPOT.TB (series test) can reduce misdiagnosis rate and improve diagnosis accuracy. In the differential diagnosis of SNPT and pneumonia, percentage of M and $\mathrm{ML}$ ratio combined with T-SPOT.TB (series test) can reduce misdiagnosis rate and improve diagnosis accuracy.

\section{Declarations}

\section{Abbreviations}

M: Monocytes; L: Lymphocytes; ML ratio: ratio of Monocytes to Lymphocytes; SNPT: Smear Negative Pulmonary Tuberculosis; HC: Healthy Control; CAP: Community-Acquired Pneumonia; HAP: Hospital- 
Acquired Pneumonia; NTM: Non-Tuberculous Mycobacterium; ROC: Receiver Operating Characteristic; HIV: Human Immunodeficiency Virus; TST: Tuberculin Skin Test; TB-Ab: Tuberculosis Antibody; IGRAs: Interferon-y Release Assays.

\section{Ethics approval and consent to participate}

The protocols applied in this study were approved by the Ethics Committee of Beijing Chest Hospital, Capital Medical University. Informed consent was waived due to the retrospective study design.

\section{Consent for publication}

Not applicable.

\section{Availability of data and material}

The datasets used and/or analyzed during the current study are available from the corresponding author on reasonable request.

\section{Competing interests}

Dr. Zhang serves as an associate editor to BMC infectious diseases, but he declares that he has no competing interests.

\section{Funding}

The design of the study was supported by Research and Evaluation of New Protocols and Technologies for the Treatment of Drug-resistant Tuberculosis (2018ZX10722301). The collection of the study was supported by the Beijing Natural Science Foundation (7192038 and 7164245) and National Natural Science Foundation (81902024), the Beijing Nova Program (Z161100004916080). The analysis of the study was supported by the National Science and Technology Major Project of China (2015ZX10004801003 and 2017ZX10201301-004) and the 'Beijing Municipal Administration of Hospitals' Youth Programme (QML20151501). The interpretation of data of the study was supported by the Tongzhou Yunhe Project (YH201807, YH201903 and YH201921). Writing the manuscript of the study was supported by the 'Beijing Municipal Administration of Hospitals' Ascent Plan (DFL20181601).

\section{Authors' contributions}

XL: study design, analysis of results, manuscript preparation. LP, ZD and QL: study design, analysis of results, critical review and modification of the manuscript. SS, HY, ZH, WS and WL: statistical analysis and critical review of the manuscript. All authors reviewed and approved the final version of the manuscript.

\section{Acknowledgments}

Not applicable. 


\section{Author details}

${ }^{1}$ Beijing Key Laboratory for Drug Resistant Tuberculosis Research, Beijing Tuberculosis and Thoracic Tumor Research Institute, Beijing Chest Hospital, Capital Medical University, No. 97, Machang, Tongzhou District, Beijing 101149, China.

\section{References}

1. China NHCotPsRo: Health industry standard of the People's Republic of China-“Classification of tuberculosis (WS196-2017)". 2017.

2. Technical Guidance Group of the Fifth National TB Epidemiological Survey TOotFNTES: The fifth national tuberculosis epidemiological survey in 2010. Chin J Antituberc 2012, 34(8):485-508.

3. Huang Fei LE, Xia Yinyin, Du Xin, Ma Yan, Cheng Shiming: The analysis of national smear negative pulmonary tuberculosis incidence and mortality, 2005-2014. Chin J Antituberc 2015, 37(5):473-477.

4. Horne DJ, Kohli M, Zifodya JS, Schiller I, Dendukuri N, Tollefson D, Schumacher SG, Ochodo EA, Pai M, Steingart KR: Xpert MTB/RIF and Xpert MTB/RIF Ultra for pulmonary tuberculosis and rifampicin resistance in adults. Cochrane Database Syst Rev 2019, 6:CD009593.

5. Sampath P, Moideen K, Ranganathan UD, Bethunaickan R: Monocyte Subsets: Phenotypes and Function in Tuberculosis Infection. Front Immuno/ 2018, 9:1726.

6. Lu Xiwei WJ, Liu Jinghua, et al: Comparing analysis between chest X-ray film and CT scan in the diagnosis of smear negative pulmonary tuberculosis. Chin J Antituberc 2008, 30(4):283-287.

7. China NHCotPsRo: Health industry standard of the People's Republic of China-"Diagnosis for pulmonary tuberculosis (WS288-2017)". 2017.

8. Li Linyang CL, Li Qi: Diagnosis value of tuberculosis infection of T cells spot test assay in pulmonary tuberculosis. Chin J Lung Dis (Electronic Edition) 2014, 7(6):32-36.

9. Detjen AK, Keil T, Roll S, Hauer B, Mauch H, Wahn U, Magdorf K: Interferon-gamma release assays improve the diagnosis of tuberculosis and nontuberculous mycobacterial disease in children in a country with a low incidence of tuberculosis. Clin Infect Dis 2007, 45(3):322-328.

10. Tuberculosis branch of Chinese medical association CJoTaRDeb: Recommendations for the application of interferon-y release assays in China. Chin J Tuberc Respir Dis 2014, 37(10):744-747.

11. Naranbhai V, Fletcher HA, Tanner R, O'Shea MK, McShane H, Fairfax BP, Knight JC, Hill AV: Distinct Transcriptional and Anti-Mycobacterial Profiles of Peripheral Blood Monocytes Dependent on the Ratio of Monocytes: Lymphocytes. EBioMedicine 2015, 2(11):1619-1626.

12. La Manna MP, Orlando V, Dieli F, Di Carlo P, Cascio A, Cuzzi G, Palmieri F, Goletti D, Caccamo N: Quantitative and qualitative profiles of circulating monocytes may help identifying tuberculosis infection and disease stages. PLoS One 2017, 12(2):e0171358.

13. Sumaira Iqbal UA, Syed Badshah Hussain Zaidi: Monocyte Lymphocyte Ratio as a Possible Prognostic Marker in Antituberculous Therapy. Journal of Rawalpindi Medical College (JRMC) 2014, 
18(2):178-181.

14. Wang W, Wang LF, Liu YY, Yang F, Zhu L, Zhang XH: Value of the Ratio of Monocytes to Lymphocytes for Monitoring Tuberculosis Therapy. Can J Infect Dis Med Microbio/ 2019, 2019:3270393.

15. Sibley L, Gooch K, Wareham A, Gray S, Chancellor A, Dowall S, Bate S, Marriott A, Dennis M, White AD et al: Differences in monocyte: lymphocyte ratio and Tuberculosis disease progression in genetically distinct populations of macaques. Sci Rep 2019, 9(1):3340.

16. Miyahara R, Piyaworawong S, Naranbhai V, Prachamat $P$, Kriengwatanapong $P$, Tsuchiya N, Wongyai J, Bupachat S, Yamada N, Summanapan S et al: Predicting the risk of pulmonary tuberculosis based on the neutrophil-to-lymphocyte ratio at TB screening in HIV-infected individuals. BMC Infect Dis 2019, 19(1):667.

17. Rakotosamimanana N, Richard V, Raharimanga V, Gicquel B, Doherty TM, Zumla A, Rasolofo Razanamparany V: Biomarkers for risk of developing active tuberculosis in contacts of TB patients: a prospective cohort study. Eur Respir J 2015, 46(4):1095-1103.

18. Mu Chuanyong HJ: Progress of immune cells in tumor microenvironment and immune esccape of lung cancer. Int J Respir 2014, 34(4):261-264.

19. Li Xiangwei JQ, Gao Lei: A short view on the epidemiology of latent tuberculosis infection in China. Electronic Journal of Emerging Infection Disease 2017, 2(3):146-150.

\section{Tables}

Table 1. Expression characteristics of monocytes related indicators and T-SPOT.TB in SNPT

\begin{tabular}{|c|c|c|c|c|c|c|}
\hline atection indicator & $\begin{array}{l}\text { HC group } \\
(\mathrm{N}=117)\end{array}$ & $\begin{array}{c}\text { lung cancer group } \\
(\mathrm{N}=56)\end{array}$ & $\begin{array}{c}\text { pneumonia group } \\
(\mathrm{N}=95)\end{array}$ & $\begin{array}{l}\text { SNPT group } \\
(\mathrm{N}=134)\end{array}$ & $\mathrm{F} / \mathrm{C}^{2}$ test & $P$ value \\
\hline rcentage of M & $7.62 \pm 1.97$ & $7.06 \pm 1.34$ & $8.17 \pm 2.13^{*}$ & $8.96 \pm 2.46^{\& \S £}$ & $13.841^{\mathrm{a}}$ & 0.001 \\
\hline$\%, x \pm S)$ & $34.76 \pm 7.15$ & $26.46 \pm 8.04 @$ & $30.57 \pm 10.70^{\# *}$ & $27.90 \pm 8.95^{\& f}$ & $16.854^{\mathrm{a}}$ & 0.001 \\
\hline $\begin{array}{l}\text { L ratio } \\
x \pm S)\end{array}$ & $0.23 \pm 0.08$ & $0.30 \pm 0.13^{@}$ & $0.31 \pm 0.18^{\#}$ & $0.36 \pm 0.19^{\& \S £}$ & $15.697^{\mathrm{a}}$ & 0.001 \\
\hline $\begin{array}{l}\text { SPOT.TB } \\
\text { ase(\%)] }\end{array}$ & & & & & $200.557^{\mathrm{b}}$ & 0.001 \\
\hline sitive & $0(0.00)$ & 18(32.10)@ & $35(36.80)^{\#}$ & $117(87.30)^{\& \S E}$ & & \\
\hline egative & $117(100.00)$ & $38(67.90)$ & $60(63.20)$ & $17(12.70)$ & & \\
\hline
\end{tabular}

Notes $\square$ M: monocytes, L: lymphocytes, ML ratio: ratio of monocytes to lymphocytes. $a=F, b=c^{2}$. @ : The $P$ value of HC group and lung cancer group was less than 0.05 , \#: The $P$ value of HC group and pneumonia group was less than $0.05, \&$ : The $P$ value of HC group and SNPT group was less than 0.05 , *: The $P$ value of lung cancer group and pneumonia group was less than 0.05 ,§: The $P$ value of lung cancer group and SNPT group was less than 0.05, $€$ : The $P$ value of pneumonia group and SNPT group was less than 0.05. 
Table 2. Analysis of predictive probability of monocytes related indicators and TSPOT.TB in SNPT and HC

\begin{tabular}{llllll}
\hline Test result variable & AUC curve & Standard error & $P$ value & \multicolumn{2}{c}{$95 \% C I$} \\
\cline { 4 - 6 } & & & & Down & Upper \\
\hline Percentage of M prediction probability & 0.688 & 0.033 & 0.000 & 0.622 & 0.753 \\
Percentage of L prediction probability & 0.739 & 0.032 & 0.000 & 0.676 & 0.802 \\
ML ratio prediction probability & 0.791 & 0.029 & 0.000 & 0.735 & 0.847 \\
T-SPOT.TB prediction probability & 0.952 & 0.014 & 0.000 & 0.925 & 0.980 \\
$\begin{array}{l}\text { Percentage of M and T-SPOT.TB } \\
\text { prediction probability }\end{array}$ & 0.965 & 0.012 & 0.000 & 0.943 & 0.988 \\
Percentage of L and T-SPOT.TB & 0.963 & 0.012 & 0.000 & 0.939 & 0.987 \\
prediction probability & & & & \\
ML ratio and T-SPOT.TB prediction probability & 0.975 & 0.009 & 0.000 & 0.957 & 0.994 \\
\hline
\end{tabular}

Table 3. The diagnostic efficacy of monocytes related indicators and T-SPOT.TB in SNPT and $\mathrm{HC}$

\begin{tabular}{|c|c|c|c|c|c|}
\hline & $\begin{array}{c}\text { Sensitivity } \\
\text { (\%) }\end{array}$ & $\begin{array}{c}\text { Specificity } \\
(\%)\end{array}$ & $\begin{array}{l}\text { Misdiagnosis } \\
\text { rate(\%) }\end{array}$ & $\begin{array}{l}\text { Missed } \\
\text { diagnosis } \\
\text { rate(\%) }\end{array}$ & $\begin{array}{l}\text { Diagnostic } \\
\text { accuracy (\%) }\end{array}$ \\
\hline itage of $\mathrm{M}$ & $70.15(94 / 134)$ & 58.97(69/117) & $41.03(48 / 117)$ & $29.85(40 / 134)$ & $64.94(163 / 251)$ \\
\hline ıtage of L & $79.85(107 / 134)$ & $52.99(62 / 117)$ & $47.01(55 / 117)$ & $20.15(27 / 134)$ & $67.33(169 / 251)$ \\
\hline io & $80.60(108 / 134)$ & 60.68(71/117) & $39.32(46 / 117)$ & $19.40(26 / 134)$ & $71.31(179 / 251)$ \\
\hline Г.ТВ & $87.31(117 / 134)$ & $100.0(117 / 117)$ & $0.0(0 / 117)$ & $12.69(17 / 134)$ & $93.23(234 / 251)$ \\
\hline $\begin{array}{l}\text { itage of } \mathrm{M} \text { and } \mathrm{T} \text { - } \\
\Gamma \mathrm{B} \\
\text { el test } \square\end{array}$ & $97.76(131 / 134)$ & $58.97(69 / 117)$ & $41.03(48 / 117)$ & $2.24(3 / 134)$ & $79.68(200 / 251)$ \\
\hline $\begin{array}{l}\text { Itage of } \mathrm{L} \text { and } \mathrm{T} \text { - } \\
\text { ГB } \\
\text { el test } \square\end{array}$ & $97.76(131 / 134)$ & $52.99(62 / 117)$ & $47.01(55 / 117)$ & $2.24(3 / 134)$ & $76.89(193 / 251)$ \\
\hline $\begin{array}{l}\text {;io and T-SPOT.TB } \\
\text { el test! }\end{array}$ & $98.51(132 / 134)$ & $60.68(71 / 117)$ & $39.32(46 / 117)$ & $1.49(2 / 134)$ & $80.88(203 / 251)$ \\
\hline
\end{tabular}

Table 4. The diagnostic efficacy of monocytes related indicators and T-SPOT.TB in SNPT and lung cancer patients

\begin{tabular}{|c|c|c|c|c|c|}
\hline & $\begin{array}{c}\text { Sensitivity } \\
(\%)\end{array}$ & $\begin{array}{c}\text { Sensitivity } \\
(\%)\end{array}$ & $\begin{array}{l}\text { Misdiagnosis } \\
\text { rate (\%) }\end{array}$ & $\begin{array}{c}\text { Missed diagnosis } \\
\text { rate (\%) }\end{array}$ & $\begin{array}{l}\text { Diagnostic } \\
\text { accuracy (\%) }\end{array}$ \\
\hline itage of $\mathrm{M}$ & $70.15(94 / 134)$ & $78.57(44 / 56)$ & $21.43(12 / 56)$ & $29.85(40 / 134)$ & $72.63(138 / 190)$ \\
\hline ıtage of L & $79.85(107 / 134)$ & $16.07(9 / 56)$ & $83.93(47 / 56)$ & $20.15(27 / 134)$ & $61.05(116 / 190)$ \\
\hline ;io & $80.60(108 / 134)$ & $41.07(23 / 56)$ & $58.93(33 / 56)$ & $19.40(26 / 134)$ & $68.95(131 / 190)$ \\
\hline Г.ТВ & $87.31(117 / 134)$ & $67.86(38 / 56)$ & $32.14(18 / 56)$ & $12.69(17 / 134)$ & $81.58(155 / 190)$ \\
\hline $\begin{array}{l}\text { itage of } \mathrm{M} \text { and } \mathrm{T}- \\
\mathrm{\Gamma B}\end{array}$ & $59.70(80 / 134)$ & $91.07(51 / 56)$ & $8.93(5 / 56)$ & $40.30(54 / 134)$ & $68.95(131 / 190)$ \\
\hline $\begin{array}{l}\text { test } \square \\
\text { itage of } \mathrm{L} \text { and } \mathrm{T} \text { - } \\
\text { ГB } \\
\text {; test } \square\end{array}$ & $70.15(94 / 134)$ & $71.43(40 / 56)$ & $28.57(16 / 56)$ & $29.85(40 / 134)$ & $70.53(134 / 190)$ \\
\hline $\begin{array}{l}\text {;io and T-SPOT.TB } \\
\text {; test }\end{array}$ & $69.40(93 / 134)$ & $78.57(44 / 56)$ & $21.43(12 / 56)$ & $30.60(41 / 134)$ & $72.11(137 / 190)$ \\
\hline
\end{tabular}


Table 5. The diagnostic efficacy of monocytes related indicators and T-SPOT.TB in SNPT and pneumonia patients

\begin{tabular}{|c|c|c|c|c|c|}
\hline & $\begin{array}{c}\text { Sensitivity } \\
(\%)\end{array}$ & $\begin{array}{c}\text { Sensitivity } \\
(\%)\end{array}$ & $\begin{array}{l}\text { Misdiagnosis } \\
\text { rate (\%) }\end{array}$ & $\begin{array}{l}\text { Missed diagnosis } \\
\text { rate (\%) }\end{array}$ & $\begin{array}{c}\text { Diagnostic } \\
\text { accuracy (\%) }\end{array}$ \\
\hline itage of $\mathrm{M}$ & $70.15(94 / 134)$ & $46.32(44 / 95)$ & $53.68(51 / 95)$ & $29.85(40 / 134)$ & $60.26(138 / 229)$ \\
\hline Itage of L & $79.85(107 / 134)$ & $36.84(35 / 95)$ & $63.16(60 / 95)$ & $20.15(27 / 134)$ & $62.01(142 / 229)$ \\
\hline ;io & $80.60(108 / 134)$ & $43.16(41 / 95)$ & $56.86(54 / 95)$ & $19.40(26 / 134)$ & $65.07(149 / 229)$ \\
\hline Г.ТВ & $87.31(117 / 134)$ & $63.16(60 / 95)$ & $36.84(35 / 95)$ & $12.69(17 / 134)$ & $77.29(177 / 229)$ \\
\hline $\begin{array}{l}\text { itage of } \mathrm{M} \text { and } \mathrm{T}- \\
\Gamma \mathrm{B}\end{array}$ & $59.70(80 / 134)$ & $76.84(73 / 95)$ & $23.16(22 / 95)$ & $40.30(54 / 134)$ & $66.81(153 / 229)$ \\
\hline $\begin{array}{l}\text {; test } 1 \\
\text { itage of } \mathrm{L} \text { and } \mathrm{T} \text { - } \\
\Gamma \mathrm{B}\end{array}$ & 70.15(94/134) & $76.84(73 / 95)$ & $23.16(22 / 95)$ & 29.85(40/134) & $72.93(167 / 229)$ \\
\hline $\begin{array}{l}\text {; test } \square \\
\text {;io and T-SPOT.TB } \\
\text {; test } \square\end{array}$ & $69.40(93 / 134)$ & $75.79(72 / 95)$ & $24.21(23 / 95)$ & $30.60(41 / 134)$ & $72.05(165 / 229)$ \\
\hline
\end{tabular}

Figures
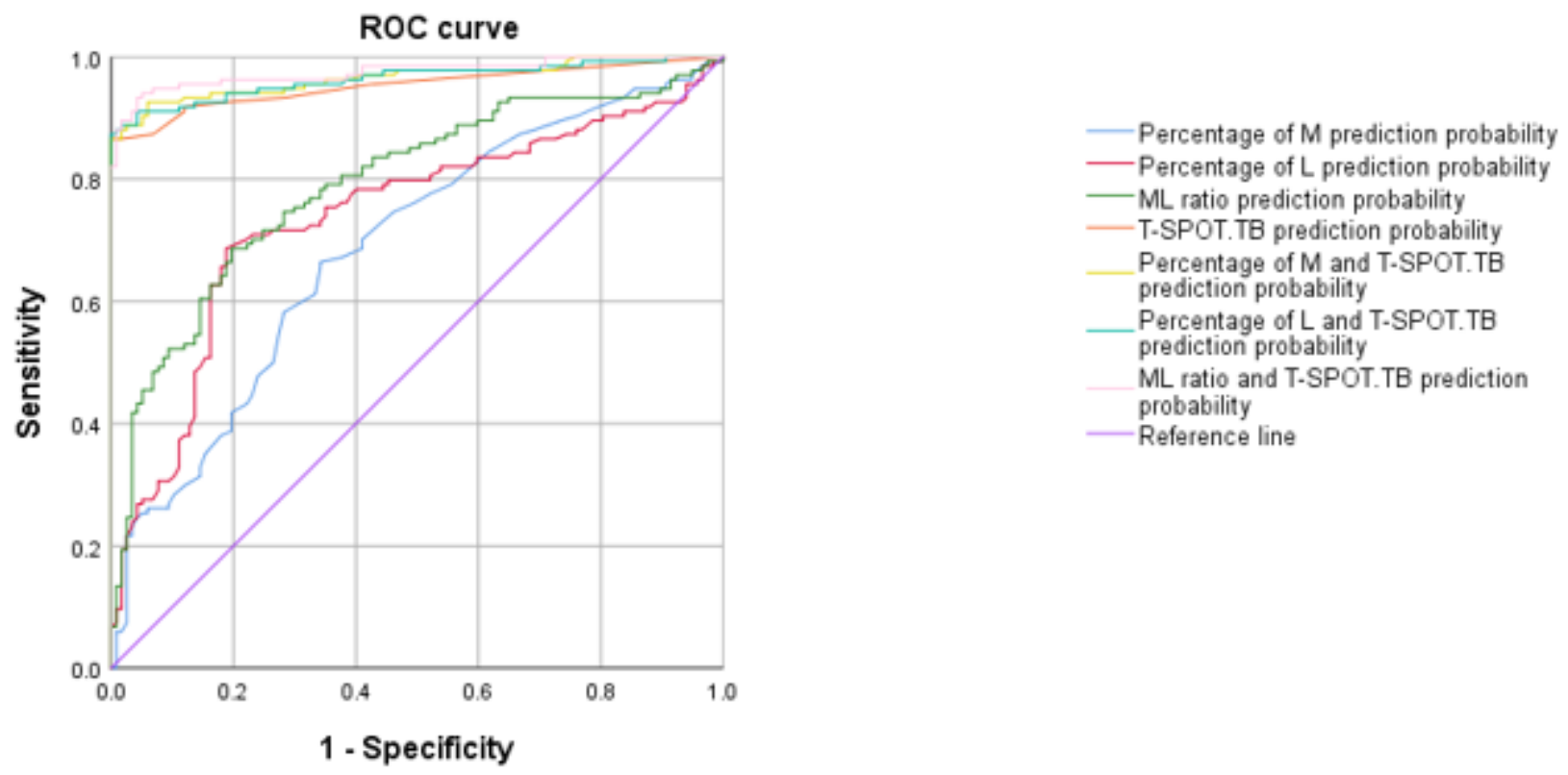

Figure 1

ROC curve of monocytes related indicators and T-SPOT.TB in SNPT and HC 